\title{
Effect of the Problem Oriented Record System on Care in a Pediatric Clinic
}

\author{
CARMI Z. MARGOLIS, JAMES L. NEWBORN, AND T. JOSEPH SHEEHAN \\ Pediatric Service, Fort Lee, Virginia and Department of Research in Health Education, University of Connecticut \\ Health Center, Farmington, Connecticut, USA.
}

\begin{abstract}
Summary
Effect of the introduction of the problem-oriented record (POR) system into a military clinic was compared with the effect of the traditional source-oriented record (SOR) on the following variables measured for appointment patients: waiting time, time spent with physician, amount of data collected, number of problems identified, data collected per min, and number of problems per min.
\end{abstract}

The POR system consisted of a standardized data base collected with the assistance of a specially trained secretary, a problem list, problem-oriented progress notes, and a biweekly peer record audit. Of 241 appointment patients seen, 60 control and 75 POR records were retrieved for grading. Records were graded according to preset criteria for amount of data collected. The typical reliability of a single grade, calculated as the intraclass correlation, was 0.78. Test retest reliability (correlation coefficient) for the principal grader was 0.95 .

Multivariate analysis of variance of the simultaneous effects of the record format (POR or SOR), patient status (new or followup), and physician on the dependent variables-amount of data collected, number of problems identified, data collected per min and number of problems identified per min-indicated that the POR system increased significantly these dependent variables. However, the POR had no effect on mean waiting time (POR 31.7 min; SOR $30.2 \mathrm{~min}$ ) or on mean time spent with the physician (POR $15.9 \mathrm{~min}$; SOR $17.1 \mathrm{~min}$ ), but did increase time spent with followup patients and decreased time spent with new patients. New patients had significantly more data collected, more problems identified, and more time spent with them than did followup patients. Change in physician only affected the amount of time spent with the patient.

It was concluded that in a military clinic the POR system can increase the number of problems identified, and the amount of data collected, without increasing physician time spent with the patient.

Between 1916 and 1920, Codman (4) first demonstrated that surgical care improves if surgeons keep careful record abstracts that include immediate and long-term outcomes. Codman's idea fell into disuse until the mid-1950's, when Lembke (9) and others $(14,15)$, began to use consistent criteria to measure quality of care as reflected in the medical record. These studies reaffirmed the fact that the existence of a record that can be audited can improve medical care. In 1964, Weed (20) proposed that changing from a traditional source-oriented record (SOR) to a problem-oriented record (POR) system (i.e., the POR format + regular audit) would improve the process of patient care. Although the POR has been adopted widely (1), Weed's proposal has elicited much debate (5, 8 , 19). However, only a handful of studies have attempted to demonstrate whether or not introduction of the POR affects patient care $(2,3,6,7,18)$. The results of these studies are inconclusive. Whereas a few researchers suggest that the POR increases both the amount of data collected and its accessibility, and decreases unnecessary tests ordered $(2,3)$, others were unable to show either that the POR increases the rapidity of problem identification or the number of problems identified, or that it improves followup care or makes record audit more accurate or more efficient $(6,18)$. All of this research used the POR format, not the system as an independent variable. Moreover, almost all of these studies $(2,3,6,18)$ were carried out in in-patient medical settings. Recently, one component of the POR system, the problem list, has been shown to improve significantly problem followup and to facilitate coordination of care in an ambulatory setting (16, 17).

The present study attempts to determine whether or not introduction of the POR system improves the process of care in a military pediatric clinic. The study is based on the assumption that if all of a patient's problems are identified and if a standardized data base is collected without decreasing care efficiency, then better care will result. We, therefore, measured the number of problems identified, amount of data collected, waiting time, and time spent with the physician both before and after introducing the POR system.

\section{METHODS}

\section{SETTING}

The pediatric clinic at Fort Lee, Virginia, provides care for dependent children, under $14 \mathrm{yr}$ old, of active duty and retired military personnel. At the time of the study, an average of 431 children/wk were seen in the clinic. Of these, $69 \%$ were acute visits seen in the early morning and afternoon, and the remaining $31 \%$ were seen by appointment. The clinic was staffed by two military and two civilian pediatricians, who saw an average of 24 patients/day. Appointment patients were seen by the same physician during approximately $80 \%$ of their visits. All four had completed training at university training programs. Two were Board certified pediatricians, one of whom had practiced at the Fort Lee clinic for $13 \mathrm{yr}$. One pediatrician had experience using a POR in both in-patient and out-patient settings, whereas another had used the POR in an in-patient setting.

Analysis of all 4142 physician diagnoses recorded in the clinic $\log$ book over a 4-month period showed that the following eight conditions comprised $61.2 \%$ of the conditions treated: otitis media (all forms), 21\%; upper respiratory infections, 15.9\%; pharyngitistonsillitis, $7.4 \%$; bronchitis, $4.4 \%$; undefined viral syndrome, $3.3 \%$; injuries, $3.3 \%$, asthma, $3 \%$; gastroenteritis, $2.9 \%$. The frequency of these eight conditions in our clinic is similar to their frequency in United States children (10).

\section{POR SYSTEM}

The format of the POR used is described in detail elsewhere (11). It consisted of past medical history and family self-history questionnaires, a routine health care flow sheet (tick sheet) based 
on the recommendations of the American Academy of Pediatrics (Fig. 1), a Problem Status Index (Fig. 2), and problem-oriented progress notes. The self-history forms were mailed to parents in advance of the child's visit, and were completed with the assistance

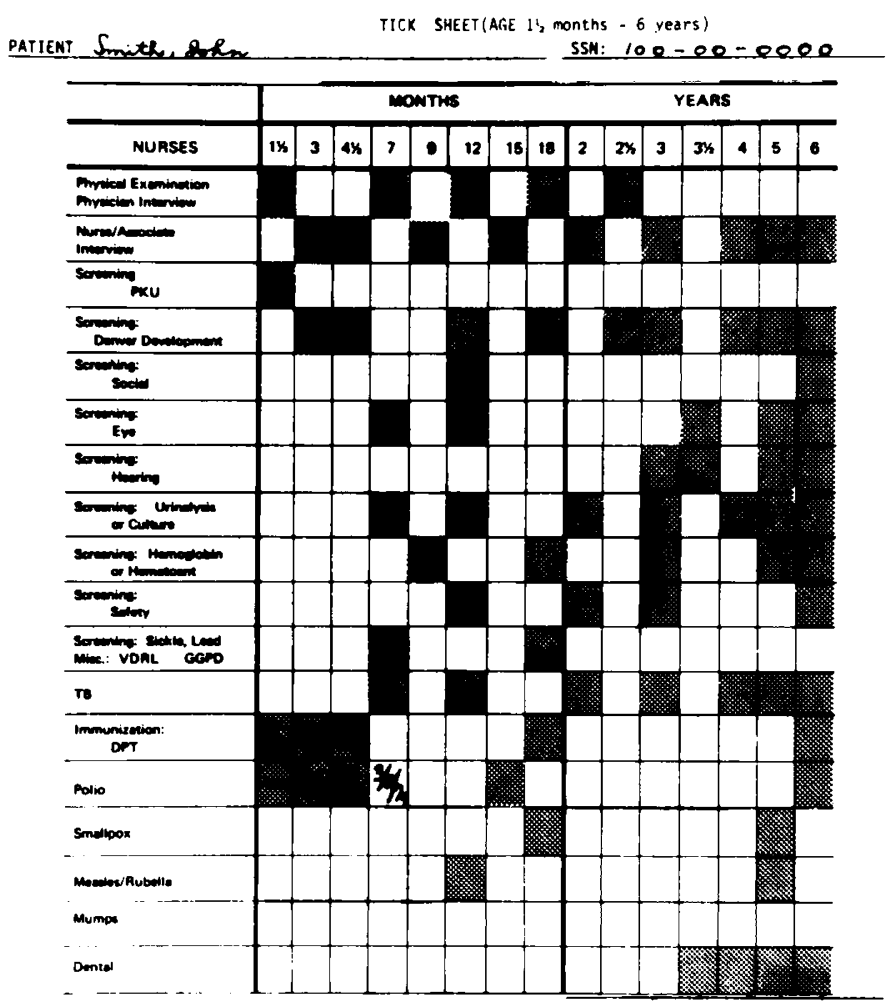

NOTE: When a procedure has been completed,enter the date in the box appropriate for

flow chart reprodu:ed courtesy of Dr. J. Aronson and MEDDAC.FT. Lee, Va.

Fig. I. Tick sheet (age 1/2 months-6 yr) of a specially trained secretary. The flow sheet was completed by the secretary before and after the child saw the physician. The Problem Status Index and progress notes were completed by the physician after he had reviewed the old chart, the questionnaires, the flow sheet, and had performed a physical examination. During the study, by special arrangement, all records that were not in transit to other clinics or military installations were filed in the pediatric clinic, in order to minimize record loss and insure proper POR format. After the POR was introduced, physicians audited all of each other's records biweekly for proper chart order, completeness of problem lists, and appropriateness of care as reflected in problem-oriented progress notes. During the control period, physicians did not audit each others records. Of the four pediatricians, only the senior author was involved in the design and implementation of the study.

\section{EXPERIMENTAL DESIGN}

Preparation for POR implementation included the following activities that were carried out over a 6 -month period: obtaining administrative approval, designing and printing new forms, hiring a POR secretary, and educating clinic physicians, nurses, and aides. Much of the process of educating physicians was accomplished by means of a self-instructional manual (12).

On February 20,1974, the POR format became the sole record format in the pediatric clinic. For 2 wk before POR introduction the following data were gathered for all appointment patients: waiting time, time spent with physician, measured by specially trained nonparticipant observers, amount of subjective and objective data collected, and number of problems identified. Three months after the POR was introduced, the same parameters were again measured for 2 wk for all appointment patients. Short data collection periods were necessitated by conditions in the clinic that were beyond the control of the investigators.

\section{RECORD AUDIT}

In order to determine the amount of data collected, all records were graded by one of the authors (C. Z. M.), using a modification PROBLEM STATUS INDEX

PATIENT Smith, John SSM: $100-00-0000$

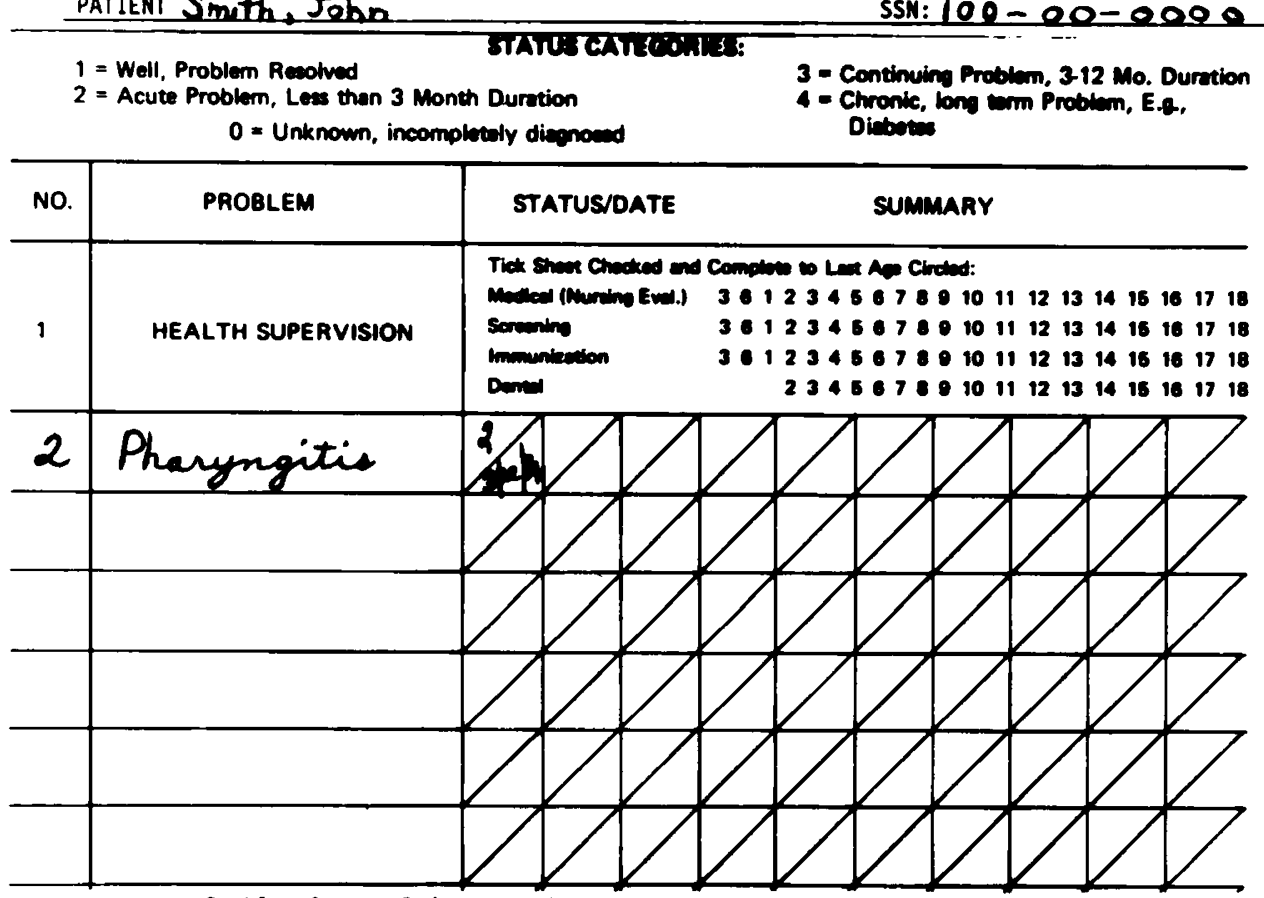

Problem Status Index reproduced courtesy Or. J. Aronson and MEDDAC, FT Lee, Va.

Fig. 2. Problem status index 
of a method discussed elsewhere for grading the POR's of 3rd yr pediatric clinical clerks (13). When clerk's records were graded, defined sections of their records were given full credit only if all preset criteria were present. For example, if all of the following three criteria were described under chief complaint-in the patient's or parent's words, onset, and duration-it was graded 3; otherwise, it was graded 0 . The major modification for grading practitioners' records was that if any criteria were recorded, full credit for the section was given. For example, if only its duration was recorded, the chief complaint was graded 3 . Thus, it was assumed that if an experienced clinician records information related to a defined section of the history or physical examination, he, in fact, has performed all the tasks defined in the preset criteria. Although it is not known whether or not this assumption is true, it tends to increase a practitioner's SOR grade, thus decreasing the difference between SOR and POR grades. The maximum achievable grade was: history, 67 points; physical examination, 21 points. (complete grading criteria available on request from C. Z. M.).

An estimate of the interrater reliability was obtained by computing the intraclass correlation, using seven records, six SOR and one POR, graded by seven raters: two pediatric faculty members and four senior house officers from the Medical College of Virginia, and the principal investigator. More SOR records were used for assessing reliability, because they were less legible, more fragmented, and, therefore, harder to grade. Test retest reliability was estimated by calculating Pearson product moment correlation coefficients for pairs of grades given by the principal investigator to the same 14 records (seven POR, seven SOR) 6 months apart.

\section{STATISTICS}

The statistical model (Fig. 3) was a three-way multivariate analysis of variance (MANOVA) to examine simultaneously the effects of the POR, patient status (new or followup), and physician upon the following five dependent variables: 1) subjective data, 2) objective data, 3) total data, 4) number of problems, and 5) time spent with physician. Effects of the three independent variables on data/time and problem/time, ratios that were calculated from the first five variables, were analyzed separately.

\section{RESULTS}

Waiting time and time spent with physician were measured for all 241 appointment patients seen during the control and experi- mental periods. However, only 158 records $(65.5 \%), 77$ control and 81 experimental, were retrieved for review and grading. The chief causes for record loss were that some records were kept by their owners against regulations, and that many Fort Lee medical records are in transit, either with personnel attending the United States Army Quartermaster School, or with personnel transferring to other assignments. Of the 158 records graded, 24 (7 experimental and 17 control) were not clear enough to allow for grading of subjective and objective data, and produced incomplete data that could not be used in the MANOVA.

\section{RELIABILITY OF THE MODIFIED GRADED PROBLEM ORIENTED RECORD}

The intraclass correlation for seven raters rating the same set of records was 0.78 . This may be interpreted as the typical reliability of a single rating. The intraclass correlation for house staff was 0.84 , whereas that for the two faculty raters and the principal grader was 0.74 . The lower correlation was explained by the consistently higher grades of the principal grader. Correlation between the two faculty raters was 0.97 .

The test retest reliability for the principal grader was 0.95 .

\section{MULTIVARIATE ANALYSIS OF VARIANCE}

Table 1 shows the combined means of the first five dependent variables for the SOR and POR, and also gives the least squares statistical estimate of the differences between means. This multivariate $F$ ratio indicates that introduction of the POR system had a highly significant effect. This effect is distinct from any effect due to differences among physicians, or patient status. Univariate $F$ ratios indicate that this effect is highly significant for subjective data, objective data, total data, and number of problems identified, but not for time.

Table 2 shows the combined means for followup patients and new patients. Again, the multivariate $F$ ratio indicates that the patient status has a highly significant effect on the dependent variables. This effect is distinct from any effect due to record system or physicians. Univariate $F$ ratios indicate that significantly more data are collected, more problems are identified, and more time is spent with new as compared with followup patients.

The multivariate $F$ ratio for effects of physician on the dependent variables is significant $(\mathrm{F}=2.16, P<0.007$, df 15,315$)$, but the only significant univariate $F$ ratio is for time. Thus, physicians seemed to vary significantly only in time spent with patient.

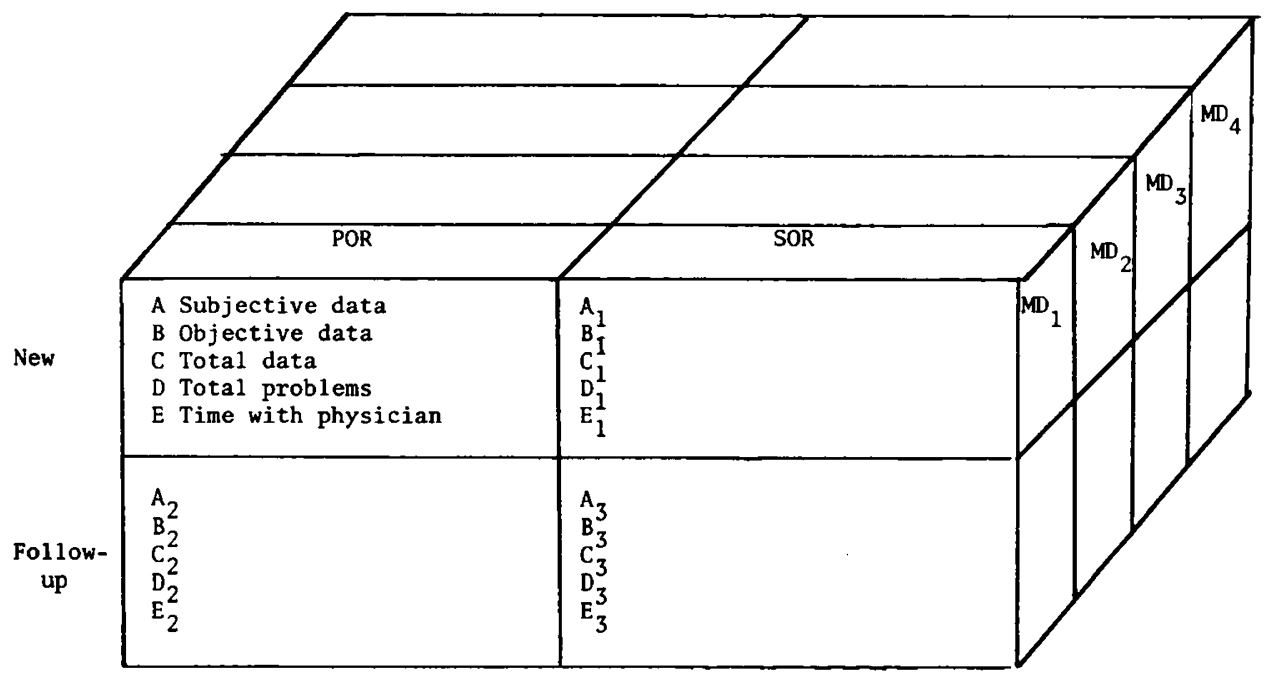

\footnotetext{
A - E are the dependent variables. The independent variables are patient status (new or followup), record format (POR or SOR), and physician $\left(\mathrm{MD}_{1-4}\right)$
}

Fig. 3. Model of multivariate analysis of variance used in the POR study 
Table 1. Estimated combined means for SOR and POR with least squares estimates of effects

\begin{tabular}{lcrcccc}
\hline & $n$ & History & Physical & Data & $\begin{array}{c}\text { No. of } \\
\text { problems }\end{array}$ & $\begin{array}{c}\text { Time } \\
(\mathrm{min})\end{array}$ \\
\hline SOR & 60 & 5.66 & 6.58 & 12.64 & 1.28 & 15.12 \\
POR & 74 & 26.40 & 8.82 & 40.67 & 3.51 & 13.76 \\
Effects' & & & & & \\
\hline & $+20.73^{2}$ & $+2.24^{3}$ & $+28.03^{2}$ & $+2.22^{2}$ & $-1.36(\mathrm{NS})$ \\
\hline
\end{tabular}

Table 2. Estimated combined means for patient status and least square estimates of effects

\begin{tabular}{lcccccc}
\hline & $n$ & History & Physical & Data & $\begin{array}{c}\text { No. of } \\
\text { problems }\end{array}$ & $\begin{array}{c}\text { Time } \\
(\mathrm{min})\end{array}$ \\
\hline $\begin{array}{l}\text { Followup } \\
\text { patient }\end{array}$ & 65 & 15.16 & 4.87 & 23.99 & 2.36 & 12.33 \\
$\begin{array}{l}\text { New } \\
\text { patient }\end{array}$ & 69 & 23.81 & 11.28 & 38.67 & 3.18 & 16.10 \\
Effects $^{1}$ & & $+8.64^{2}$ & $+6.41^{2}$ & $+14.68^{2}$ & $+.82^{3}$ & $+3.77^{4}$ \\
\hline
\end{tabular}

' Multivariate $\mathrm{F}$ ratio with 5 and $114 \mathrm{df}$ is $13.47(P<0.0001)$.

${ }^{2} P<0.0001$.

${ }^{3} P<0.007$.

${ }^{4} P<0.03$.

Table 3. Observed means for SOR and POR in combination with patient condition

\begin{tabular}{lcccccc}
\hline & $n$ & History $^{2}$ & Physical & Data $^{3}$ & $\begin{array}{c}\text { No. of } \\
\text { problems }\end{array}$ & $\begin{array}{c}\text { Time }^{2} \\
\text { (min) }\end{array}$ \\
\hline $\begin{array}{l}\text { SOR } \\
\begin{array}{l}\text { Followup } \\
\text { patients }\end{array}\end{array}$ & 27 & 3.54 & 3.38 & 7.60 & 1.05 & 11.14 \\
$\begin{array}{l}\text { New } \\
\text { patients }\end{array}$ & 23 & 7.09 & 9.48 & 17.00 & 1.22 & 19.61 \\
$\begin{array}{l}\text { POR } \\
\text { Followup }\end{array}$ & 28 & 19.46 & 5.14 & 30.61 & 2.86 & 14.54 \\
$\begin{array}{l}\text { patients } \\
\text { New }\end{array}$ & 46 & 31.87 & 12.14 & 49.30 & 4.22 & 15.02 \\
patients & & & & & & \\
\hline
\end{tabular}

\footnotetext{
${ }^{1}$ Multivariate $\mathrm{F}$ ratio with 4 and $114 \mathrm{df}$ is $4.13(P<0.0018)$.

${ }^{2} P<0.005$.

${ }^{3} P<0.02$.
}

The only significant interaction between main effects is that between record system and patient status. Table 3 gives the relevant combined means and least squares estimate. Although the POR seems to have increased time spent with followup patients, it decreased time spent with new patients.

Table 4 gives combined means and least squares estimates that demonstrate a highly significant effect of the record system on data/time and problem/time. Neither patient status nor physician affected these two variables significantly, nor were any of the interactions significant.

\section{WAITING TIME AND TIME SPENT WITH PHYSICIAN}

Mean waiting times calculated for all 113 SOR patients and all 128 POR patients were 30.2 and $31.7 \mathrm{~min}$, respectively. Mean times spent with the physician for 113 SOR's and 128 POR's were 17.1 and $15.9 \mathrm{~min}$, respectively. Neither of these differences is significant.
Table 4. Estimated combined mean for times 1 and 2 with least squares estimates of effects

\begin{tabular}{lcc}
\hline & Data/time & Problems/time \\
\hline SOR & 98.89 & 13.91 \\
POR & 305.90 & 28.58 \\
& & \\
Effects $^{1}$ & $+207.01^{2}$ & $+14.67^{3}$ \\
\hline
\end{tabular}

\footnotetext{
${ }^{1}$ Multivariate $\mathrm{F}$ ratio with 2 and $117 \mathrm{df}$ is $20.12(P<0.0001)$

${ }^{2} P<0.0001$.

${ }^{3} P<0.0004$
}

\section{DISCUSSION}

In general, the effects of the POR format have been tested using two methods. The first method has been to compare the POR with the SOR in a classroom setting. Bertucci et al. (3) compared problem-oriented progress notes written by 15 nurses in response to five standardized case histories with traditional notes written in response to the same case histories by another 15 nurses. Results showed that problem-oriented progress notes contained significantly more subjective data, but did not contain more objective data, better assessments, or better plans. Fletcher (6) adapted case histories of four patients with complex general medical problems to equivalent POR and SOR formats. He reported that house officers could not audit the POR format faster or more accurately than the SOR format. A closer look at his data indicates that house officers work faster with the system they were accustomed to using before the experiment: residents from Hopkins were better at the SOR, whereas residents from Baltimore City were better at the POR. A major methodologic problem of the above two studies is that a classroom test of POR effectiveness may severely limit the generalizability of the results to real settings. Fletcher (6), for example, exerted maximum control over his variables, but his results might not be the same if the study were performed on a hospital ward.

The second method used to test Weed's proposal (20) has been to determine whether or not the process of patient care improves after introduction of the POR. Froom (7) demonstrated that conversion to a POR format can be effected efficiently in an established solo family practice. However, Froom's statements regarding the superiority of the POR for improving data collection and problem identification can only be accepted as impressions, because he did not carry out a controlled comparison of POR's and SOR's. Switz (18) compared two groups of patients discharged from a medical ward with the diagnosis of anemia before and after introduction of the POR. He could not show any difference between the groups in completeness of the history and physical examination, in number of days before problem identification, in treatment, or in problem resolution. Aranda (2) studied the effect of the POR on problem identification, followup care, and filing of laboratory data in records of patients on the internal medicine service of a military community hospital. He could not show any difference before and after introduction of the POR in number of problems identified, or in quality of followup care, but he found that laboratory data were more accessible, and unnecessary tests were done less frequently after POR introduction.

Simborg et al. (16) and Starfield et al. (17) studied the effect of POR format on ambulatory care. Simborg et al. (16) demonstrated that a problem list increased the followup rate of problems appearing on the list. Followup rate increased regardless of problem type (diagnosis or symptom), practitioner type (pediatrician or internist), and practitioner continuity (prepaid or inner city clinic). Progress note format was not shown to affect followup rate. Starfield et al. (17) demonstrated that a minirecord, consisting of a problem list that also listed therapies and visits to other clinics, facilitated, but did not improve, coordination of care. The minirecord improved practitioner recognition of problems and therapies previously listed in the same, but not in other clinics. 
A difference in method between the present study and previous ones is that the authors chose the POR system, which includes record audit, as our independent variable, whereas previous studies have studied only POR format. Starfield and coworkers (16, 17) have studied two components of POR format: the problem list and problem-oriented progress notes. The authors chose the POR system for two reasons. First, Weed's (20) statement about the positive effect of the POR on care has always assumed that POR audit is introduced with POR format. Second, it was felt that because no component of the POR had as yet been shown definitely to affect care, first, the entire POR system would be tested, and then, if an effect was obtained, POR audit and POR format would be tested separately. A limitation of the present study, therefore, is that any POR effect demonstrated may have been produced by POR audit, rather than by POR format.

Only two of the studies reviewed $(16,17)$ attempt to demonstrate whether ambulatory care is improved by introduction of the POR. Our data show that after the POR system was introduced, pediatricians working in a nonteaching clinic collected significantly more data and identified significantly more problems in the same amount of time. Significantly, more data were collected from new patients in significantly less time. Moreover, waiting time remained the same after POR introduction. We cannot conclude from this study that identified problems are followed up. However, if the conclusions of Simborg's et al. (16) are correct, then they have a better chance of being followed up than problems not appearing on a problem list.

Two sources of possible bias in our study were record loss and short data collection periods. We were unable to select our records either randomly or consecutively, but we felt it reasonable to assume that the two chief causes of record loss, transit of personnel, and illegibility, affected the control and experimental groups equally. Short data collection periods affect the interpretation of the data in two ways. First, because our samples are small, they may not be representative. Second, we cannot conclude that any effect of the POR persisted beyond the first 3 months.

Increase in data collected was due chiefly to the self-history questionnaires, which reviewed the complete past medical and family history and were scored 30 points. Although the secretary facilitated the process of completing these questionnaires, her own data collection, as reflected in the tick sheet, was graded only 1 point. Another important source of data was the old record. All pediatricians admitted that until they were required to construct a complete problem list, they rarely reviewed the entire old record even when seeing new patients. In the present study, we did not exclude the possibility that a pediatrician using the SOR and also reviewing the entire old record, would identify as many problems as a pediatrician using the POR system. However, we would hypothesize that the number of problems identified was a function not only of reviewing the old record, but rather of an interaction between the necessity to construct a complete problem list and information in the old record.

In order to demonstrate whether the number of problems identified depended upon the amount of data collected, the correlations between the number of problems and history, physical, data, and time spent with physician were examined. They were 0.36 (history), 0.16 (physical), 0.32 (data), and 0.18 (time), respectively, all moderate to low. It does not appear, therefore, that the number of problems identified depends heavily upon the amount of data gathered. The weak correlation between amount of data collected and number of problems identified at first seemed surprising. It can probably be explained, however, both by the large number of instances in which many problems were elicited by the experienced pediatrician with few questions and a brief physical, and by those instances in which a complete history and physical examination yielded only one problem: i.e., "routine health care." Although an increase in data collected may not lead to an increase in number of problems identified, we do feel that an increase in data collected according to accepted standards for well child care should increase the quality of well child care.

Inasmuch as our data show a strong effect of the POR system on amount of data collected and number of problems identified, they do not agree with some of the data collected in a medical ward setting. One explanation for our positive results might be that they may have been produced by the audit component of our POR system. In order to elucidate this possibility, we are presently testing POR format and audit separately for their effects on our independent variables. An explanation for lack of POR format effect in hospital settings is that both on medical and pediatric teaching wards a strong effort has long been made on admission to do as complete a workup, and to identify as many problems as possible. In ambulatory settings, especially patient care oriented settings, efforts at standardized data collection and exhaustive problem identification are rare. We propose that it is in an ambulatory care setting that the POR system has its greatest effect.

\section{REFERENCES AND NOTES}

1. Adamson, T. E., et al: The teaching and use of the problem-oriented record in medical schools. J. Med. Ed., 49: 905 (1974)

2. Aranda, J. M.: The problem-oriented medical record: experience in a community hospital. JAMA, 229: 549 (1974)

3. Bertucci, M., Houston, M., and Perloff, E.: Comparative study of progress notes using problem-oriented and traditional methods of charting. Nurs. Res., 23: 351 (1974)

4. Codman, E. A.: A study in hospital efficiency: the first five years. (Boston, Thomas Todd Co., 1916)

5. Feinstein, A. R.: The problems of the problem-oriented record. Ann. Int. Med. 78: 751 (1973)

6. Fletcher, R. H.: Auditing problem-oriented records and traditional records. N Engl. J. Med., 290: 829 (1974)

7. Froom. J.: Conversion to problem-oriented records in an established practice: a timed study. Ann. Int. Med., 78: 254 (1973)

8. Goldfinger, S. E.: The problem-oriented record: critique from a believer. N. Engl. J. Med., 288: 606 (1972)

9. Lembke. P. A.: Medical auditing by scientific methods, illustrated by major female pelvic surgery. JAMA, 162: 646 (1956)

10. Lengthening Shadows. p. 43 American Academy of Pediatrics, (Evanstion, 1971)

11. Margolis, C. Z.: The Pediatric Problem-Oriented Record. (Docent Corporation, Pleasantville, 1977)

12. Margolis, C. Z., Sheehan, T. J., and Meyers, A.: Using a workshop to determine educational effectiveness of a text on the problem-oriented record. J. Med. Ed. 51: 767 (1976)

13. Margolis, C. Z., Sheehan, T. J., and Stickley, W. T.: A graded problem-oriented record to evaluate clinical performance. Pediatrics, $51: 980$ (1973)

14. Morehead, M. A.: The medical audit as an operational tool. Am. J. Public Health, 57: 1643 (1967)

15. Rosenfeld, L. S.: Quality of medical care in hospitals. Am. J. Public Health, 47: 856 (1957)

16. Simborg, P. W., Starfield, B. H., and Horn, S. D.: Information factors affecting problem followup in ambulatory care. Medical Care, 14: 848 (1976)

17. Starfield, B., Simborg, D., Johns, C., and Horn, S.: Coordination of care and its relationship to continuity and medical records. Med. Care, 15: 929 (1977)

18. Switz, D. M.: The problem-oriented medical record: evaluation and management of anemia before and during use. Arch. Int. Med., 136: 1119 (1976)

19. The problem of the problem-oriented record. N. Engl. J. Med. 288: I133 (1973)

20. Weed, L. L.: Medical records, patient care, and medical education. Irish J. Med. Sci., 6: 271 (1964)

21. The authors thank Col. Arren C. Buchanan and Cpt. Howard Keel, and the staff of the Pediatric Clinic at Fort Lee, Virginia, for their encouragement and support in performing the study, Drs. Jerry Friedlander and John Beck for helpful editorial criticism, and Mrs. Rita Soullam for secretarial assistance.

22. The opinions expressed herein are those of the authors, and cannot be construed as reflecting the views of the Department of the Army or of the Army Service at large.

23. Requests for reprints should be addressed to: Dr. Carmi Z. Margolis, University for Health Sciences, Primary Care Unit, Ben Gurion University of the Negev, Beersheba, Israel.

24. Received for publication March 8, 1978

25. Received for publication September 13, 1978. 